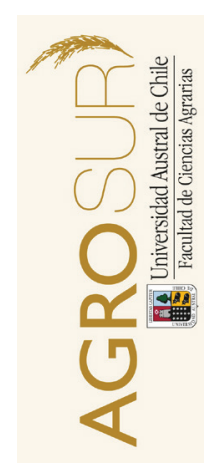

\title{
Variabilidad espacial y temporal de la disponibilidad de nutrientes y la condición de acidez de suelos volcánicos
}

\author{
Spatial and temporal variability of nutrient availability and soil acidity \\ condition in volcanic soils
}

\author{
Valle, S.R. ${ }^{a, b^{*}}$, Carrasco, J. ${ }^{a}$, Pinochet, D. ${ }^{a, b}$, MacDonald, R. (In memoriam) ${ }^{a}$ \\ ${ }^{a}$ Instituto de Ingeniería Agraria y Suelos; ${ }^{b}$ Centro de Investigación en Suelos Volcánicos (CISVo). \\ Facultad de Ciencias Agrarias. Universidad Austral de Chile. Campus Isla Teja, Valdivia, Chile.
}

\begin{tabular}{l} 
A R T I C L E I N F O \\
\hline Article history: \\
Received 29.07.14 \\
Accepted 26.01.15 \\
\hline Keywords: \\
Spatial and temporal variability \\
Volcanic soils \\
Levels \\
Chemical properties \\
\hline
\end{tabular}

Original Research Article, Soil Science

*Corresponding author:

Susana R. Valle

E-mail address: susanavalle@uach.cl

\begin{abstract}
A B S T R A C T
Spatial and temporal variability of nutrient availability and the acidity are the result of soil formation conditions, management and variation among seasons. It is important to know and consider this variability to design future management practices. Therefore, the objective of this study was to evaluate the variability of nutrient availability and the acidic condition of volcanic soils, under pasture and forest, considering different spatial and temporal scales in Máfil municipality, Los Ríos Region. Two spatial scales were considered, one macro (transect of $14 \mathrm{~km}$, with distance between samples of $100 \mathrm{~m}$ ) and a micro (sampling grid at intervals of $50 \mathrm{~m}$ ), with the seasonal variation (6 sampling dates). Soil samples were taken at $0-20 \mathrm{~cm}$ of soil depth. The acidic condition was assessed using $\mathrm{pH}\left(\mathrm{H}_{2} \mathrm{O}\right.$ and $\left.\mathrm{CaCl}_{2}\right)$, exchangeable $\mathrm{Al}$, sum of bases, ECEC and $\mathrm{Al}$ saturation. Nutrient availability in soil was assessed through mineral $\mathrm{N}$, available $\mathrm{S}$, P-Olsen and exchange cations. Main results show there were no significant differences $(p>0.05)$ between sampling scales, using conventional statistical analysis for nutrient availability and acidity parameters. However, the spatial analysis shows clear differences between the scales through the different adjustment models and types of spatial dependence. Likewise, the chemical properties showed different degrees of variation given by the sampling date. $\mathrm{N}$ and $\mathrm{S}$ available showed the highest seasonal variations.
\end{abstract}

\section{RESUMEN}

La variabilidad espacial y temporal de la disponibilidad de nutrientes y acidez están dados por las condiciones particulares de formación, manejo y variación entre las estaciones del año. Es importante conocer esta variabilidad y considerarla para diseñar futuras prácticas de manejo. Es por ello, que el objetivo de este estudio fue evaluar la variabilidad de la disponibilidad de nutrientes y de la condición de acidez de suelos de origen volcánico, bajo pradera y bosque, considerando diferentes escalas espaciales y temporales en la comuna de Máfil, Región de los Ríos. Para ello, se evaluaron dos escalas espaciales, una macro (transecto de $14 \mathrm{~km}$ aproximadamente, con distancia entre muestras de $100 \mathrm{~m}$ ) y una micro (grilla de muestreo a intervalos de $50 \mathrm{~m}$ ), en conjunto con la variación estacional ( 6 fechas de muestreo). Las muestras de suelo fueron tomadas entre 0-20 cm de profundidad. La condición de acidez se evaluó a través del pH $\left(\mathrm{H}_{2} \mathrm{O}\right.$ y CaCl 2$)$, $\mathrm{Al}$ intercambiable, suma de bases, CICE y saturación de Al. La disponibilidad de nutrientes en el suelo se evaluó a través del N mineral, S disponible, P-Olsen y bases de intercambio. Los principales resultados muestran que, con el análisis estadístico convencional no se encontraron diferencias significativas ( $p>0,05)$ entre las escalas de muestreo, para los parámetros de disponibilidad y acidez evaluados. Sin embargo, el análisis espacial muestra claras diferencias entre las escalas, a través de los diferentes modelos de ajuste y tipos de dependencia espacial. Así mismo, las propiedades químicas presentaron diferentes grados de variación dados por la fecha de muestro, donde las mayores variaciones estacionales las presentaron los parámetros de disponibilidad del $\mathrm{N}$ mineral y el S disponible.

Palabras clave: variabilidad espacial y temporal, suelos volcánicos, escalas, propiedades químicas.

\section{INTRODUCCIÓN}

Las propiedades del suelo presentan distintos grados de variabilidad espacial (Webster y Oliver, 1990; 2001). La caracterización de esta variabilidad espacial es fundamental para entender las asociaciones existentes entre las propiedades del suelo y los factores ambientales (Goovaerts, 1998). Esta variabilidad es resul- tante de la interacción de los procesos y factores de formación y, del manejo de los suelos (Jenny, 1941; Simonson, 1959); todos los cuales están sujetos a variaciones estacionales (Parkin, 1993; Cambardella et al., 1994; Timm et al., 2006; Logsdon, 2012). El reconocimiento de la importancia de esta variabilidad ha inducido su estudio, desde una escala regional a una local o predial. Por esto, la variabilidad espacial es dependiente de la 
escala de observación, de las propiedades en cuestión y de la metodología utilizada (Yemefack et al., 2005). Es así, que los estudios de clasificación de suelos tienden a utilizar una escala regional; por su parte, la variabilidad de las propiedades del suelo en escalas con un mayor nivel de detalle, a nivel predial, es dependiente de las prácticas de manejo (Yemefack et al., 2005), y es bastante desconocida y, a menudo, descrita por medios estadísticos tradicionales, en que se asume independencia de los datos (Burrough, 1993), como valores de tendencia central (promedios, modas, medianas) y de su variabilidad (desviación y error estándar, sesgo y otras) (Trangmar et al., 1985). El conocimiento de la variabilidad del suelo es fundamental para la selección de sitios uniformes y ello debe considerar las diferentes escalas de observación (Webster y Oliver, 2001; Yemefack et al., 2005; Zhang et al., 2007). Con lo cual, se pueden diseñar manejos más específicos y con menor impacto ambiental, mejorando así la eficiencia en el uso de recursos.

Los suelos de origen volcánico (Andisoles, Ultisoles e Inceptisoles), representan entre el 50-60\% de la superficie arable del país y en ellos, se desarrolla gran parte de la producción cerealera, ganadera y forestal del país (Besoain, 1985; Sadzawka et al., 2006; Bertrand y Fagel, 2008). Debido a la amplitud de usos y su importancia, en términos de producción silvoagropecuaria, es que, conocer la variabilidad de estos suelos es cada vez más relevante. Ello porque, aunque poseen un origen en común, son disímiles en muchas de sus características (Shoji et al., 1993; Dahlgren et al., 2004). Una característica común de estos suelos, es que son muy productivos, sin embargo, presentan deficiencias importantes de algunos nutrientes, principalmente de fósforo disponible (Dahlgren et al., 2004), además de serios problemas de acidez, dado principalmente por la abundante precipitación (Rowell, 1994, Sposito, 2008) en la zona sur de Chile. El objetivo de este estudio fue evaluar la variabilidad de parámetros de disponibilidad de nutrientes y de la condición de acidez de suelos de origen volcánico, considerando diferentes escalas espaciales y temporales.

\section{MATERIAL Y MÉTODO}

\section{Condiciones experimentales}

El estudio se realizó en la Provincia de Valdivia, Región de Los Ríos, durante el mes de marzo-abril del año 2011. Para evaluar la variabilidad de las escalas espaciotemporales, se utilizaron diferentes metodologías de muestreo. En primer lugar, para evaluar la variabilidad espacial a un nivel de gran escala (macro), se realizó un muestreo sobre un transecto de aproximadamente 14 km de extensión; cuyo centro, correspondió al Fundo Chile Chico, ubicado en la comuna de Máfil (Fig. 1). La distancia de muestreo fue a intervalos de $100 \mathrm{~m}$ de distancia (con un total de 242 puntos), inicialmente; sin embargo, debido a las irregularidades del terreno y la dificultad de acceso en varios puntos esta distancia fue acortada o extendida, la superficie abarcada con esta metodología fue de aproximadamente 1100 ha. Todos los puntos muestreados fueron georreferenciados en el Datum Provisorio Sudamericano 1956 (PSAD 56). Las muestras fueron tomadas a una profundidad de $0-20 \mathrm{~cm}$. Para la evaluación de la variación espacial a menor escala (micro), se muestrearon 78 puntos bajo un sistema de grilla, cuya distancia de separación fue de $50 \mathrm{~m}$, considerando una superficie de aproximadamente 182 ha. En una tercera evaluación, se estudió la variación estacional de algunas propiedades químicas de disponibilidad y de condición de acidez, en un sector reducido dentro del predio, en áreas bajo manejo de praderas y bosque (Fig. 1). Dentro de cada sector de pradera y bosque, se colectaron 4 repeticiones a una profundidad de 0-20 $\mathrm{cm}$, siendo cada una de estas repeticiones una muestra compuesta por 10 submuestras, se siguió un muestreo en zig-zag con distancia de separación entre cada punto de $20 \mathrm{~m}$. Se consideraron 6 fechas de muestreo, entre el invierno del año 2011 y la primavera del año 2012.

\section{Evaluaciones}

Las propiedades químicas consideradas en este estudio, fueron el $\mathrm{pH}$ al agua y $\mathrm{pH}$ en cloruro de calcio en una relación 1:2,5; contenido de aluminio intercambiable (extraído con $\mathrm{KCl} 1 \mathrm{M}$ ) y bases de intercambio (Ca, Mg, Ky $\mathrm{Na}$, extraídas con acetato de amonio $1 \mathrm{M} \mathrm{pH} 7,0$ ); ambos, determinados por espectrofotometría de absorción y emisión atómica; contenido de nitrógeno mineral, a través de destilación con arrastre de vapor; azufre disponible (extraído por dihidrógeno fosfato de calcio 0,01M), determinado por turbidimetría y $\mathrm{P}$ disponible medido de acuerdo al método de extracción con bicarbonato de $\mathrm{Na} 0,5 \mathrm{M}$ a pH 8,5 y posterior determinación colorimétrica del azul de molibdeno (P-Olsen). Con los resultados de las bases y Al de intercambio, se calculó la suma de bases $(\mathrm{SB}=\mathrm{Ca}+\mathrm{Mg}+\mathrm{K}+\mathrm{Na}$ ), porcentaje de saturación de $\mathrm{Al}$ $\left(\mathrm{SAl}=[\mathrm{Al} /(\mathrm{SB}+\mathrm{Al})]^{*} 100\right)$ y capacidad de intercambio catiónico efectiva (CICE= SB + Al). Todas las metodologías usadas, están descritas en Sadzawka et al. (2004).

\section{Análisis de la información}

Se realizó un análisis de estadística descriptiva de los datos (medias, mediana, mínimos, máximos, desviación estándar, error estándar, skewness y kurtosis) para cada nivel de evaluación, tanto espacial (transecto y grilla) como temporal (estaciones del año). Se realizó un ANDEVA para evaluar las diferencias entre las escalas espaciales, para los valores medios de las variables químicas y, también para evaluar las diferencias entre las estaciones del año. El análisis espacial se realizó a través de semivariogramas (Fig. 2), krigging y mapas 


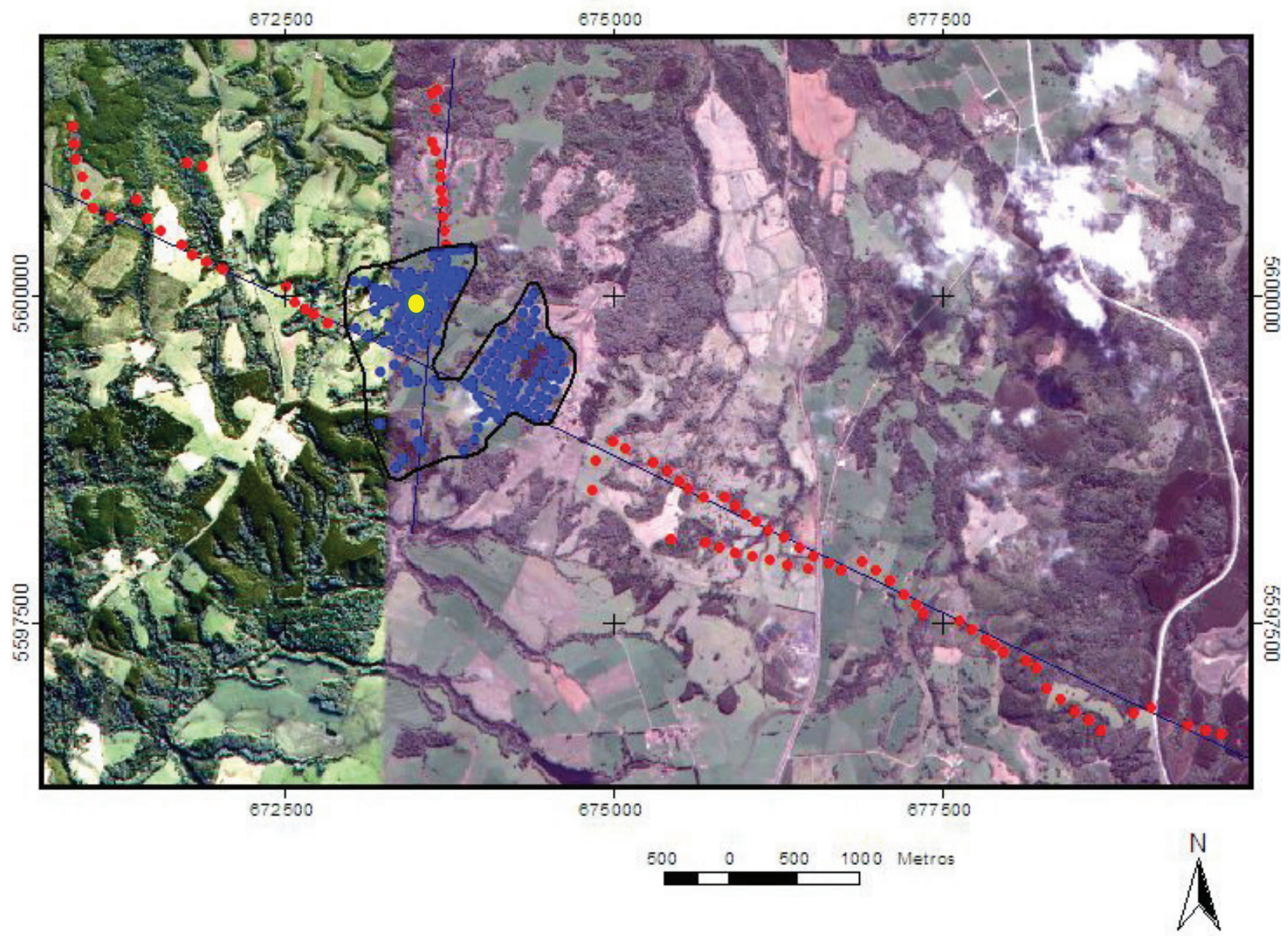

Figura 1. Ubicación de los puntos muestreados en el transecto (puntos rojos más azules), grilla (sólo puntos azules) y en la pradera y bosque donde se evaluaron los cambios estacionales (punto amarillo).

Figure 1. Location of the sampled points in the transect (blue and red dots), grid (blue dots) and the grassland and forest where seasonal changes (yellow dot) were evaluated.

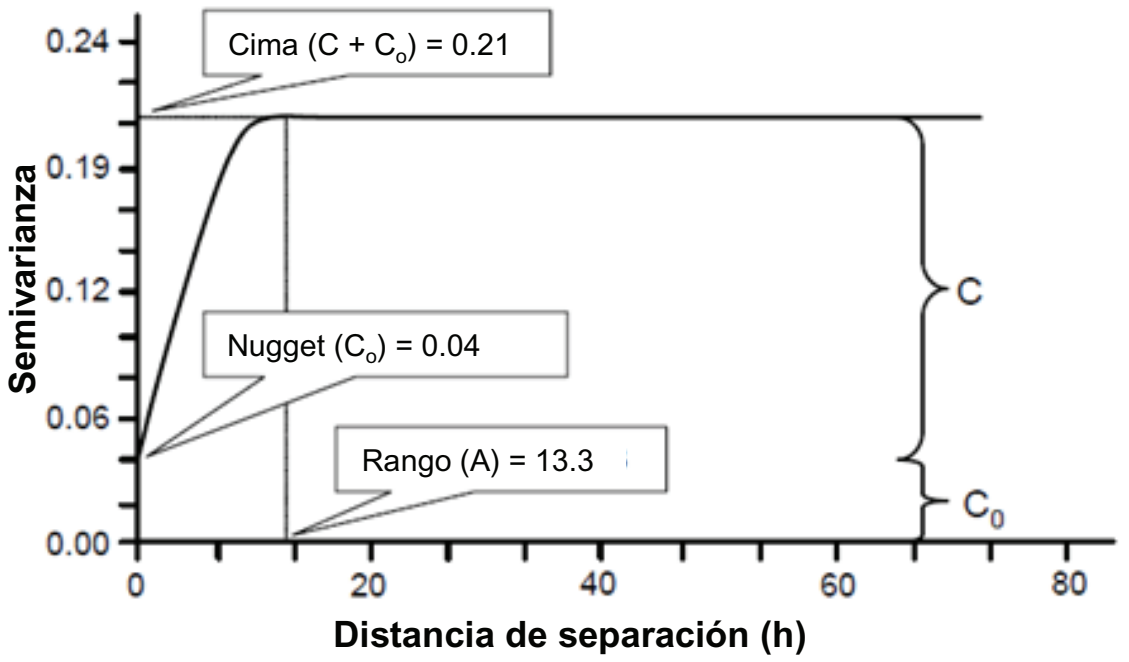

Figura 2. Esquema generalizado del semivariograma y sus parámetros descriptivos (Gamma Design Software (C)).

Figure 2. Generalized semivariogram and their descriptive parameters (Gamma Design Software (C)). 
de distribución. Dentro de los semivariogramas, los parámetros relevantes a considerar y comparar entre las escalas consideradas están, el Sill $(\mathrm{C}+\mathrm{Co})$, que es la cima, valor umbral o asíntota del modelo, que representa la varianza total; el Nugget (Co), que es el intercepto en el eje $y$ del modelo, que representa una medida del error o variabilidad de la propiedad, que no es detectada en la escala del muestreo; el Rango (A), que es la distancia de separación sobre la cual la dependencia espacial es aparente o evidente; $C$, varianza estructural; $[C /(C+C o)]$, medida de la proporción de la varianza de la muestra que es explicada por la varianza estructural (C). Para cada una de las propiedades se evaluó el grado y tipo de dependencia espacial según Cambardella et al. (1994). Estos análisis se realizaron con el software GS+ v.9 (Gamma Design Software (C).

\section{RESULTADOS Y DISCUSIÓN}

Como muestra el Cuadro 1, los valores de $\mathrm{pH}$ variaron entre 4,0 y 6,3, los valores medios para los suelos considerados en el transecto y la grilla, muestran que el pH en ambos casos es fuertemente ácido y consistente con una alta saturación de $\mathrm{Al}$, que varió entre 0,2 a $83,7 \%$. Los suelos, en general presentaron una baja dis- ponibilidad de P, S y una baja suma de bases; en cambio, se encontraron altos niveles de $\mathrm{N}$ mineral disponible (Bernier, 2000). Cuando sólo se considera los valores medios de las propiedades químicas evaluadas, se pierde mucha información, ya que éstos no muestran la variabilidad espacial de las propiedades de los suelos. Es así que, al comparar los valores de las propiedades en este estudio no se muestran diferencias significativas $(p>0,05)$ entre los valores promedios de la macro zona y la micro zona (Cuadro 1).

Al considerar el análisis espacial de las propiedades químicas, se muestra que las propiedades del suelo presentan un comportamiento diferente, representado por los diferentes ajustes de los modelos de los semivariogramas (Cuadro 2) y en los parámetros descriptores, tanto para el transecto (macro) como para la grilla (micro) de muestreo. Los modelos ajustados para algunas de estas propiedades fueron el gaussiano, esférico y exponencial, todos ellos isotrópicos; es decir, la variación estuvo dada principalmente por la distancia de separación de las muestras (Nielsen y Wendroth, 2003) con una alta dependencia espacial (Cambardella et al., 1994; Cambardella y Karlen, 1999). Cabe hacer notar que, algunos parámetros evaluados como el pH (tanto en $\mathrm{H}_{2} \mathrm{O}$, como en $\mathrm{CaCl}_{2}$ y saturación de $\mathrm{Al}$ (en grilla, micro) fueron independientes de

Cuadro 1. Estadígrafos descriptivos de las variables químicas de los suelos colectados en el Transecto-T (macrovariación) $(n=242)$ y grilla-G (microvariación) $(n=78)$.

Table 1. Descriptive statistics of the chemical variables of soils collected in the transect-T (macro variation) ( $n=242)$ and the grid (micro variation) $(n=78)$.

\begin{tabular}{lccccccccc}
\hline \multicolumn{1}{c}{ Parámetro de Suelo } & Unidad & Promedio & Mediana & Mínimo & Máximo & DE & EE & Sk & Kr \\
\hline $\mathrm{pH} \mathrm{H}_{2} \mathrm{O}$ & $\mathrm{T}$ & 5,55 & 5,56 & 4,78 & 6,29 & 0,27 & 0,02 & $-0,03$ & 0,04 \\
& $\mathrm{G}$ & 5,55 & 5,53 & 4,78 & 6,16 & 0,27 & 0,03 & $-0,10$ & 0,32 \\
$\mathrm{pH} \mathrm{CaCl}_{2}$ & $\mathrm{~T}$ & 4,86 & 4,88 & 4,02 & 5,66 & 0,31 & 0,02 & $-0,14$ & $-0,12$ \\
& $\mathrm{G}$ & 4,87 & 4,87 & 4,02 & 5,66 & 0,32 & 0,04 & $-0,14$ & 0,07 \\
P-Olsen $\left(\mathrm{mg} \mathrm{kg}^{-1}\right.$ ) & $\mathrm{T}$ & 6,62 & 5,43 & 1,16 & 43,43 & 5,00 & 0,32 & 0,07 & $-0,61$ \\
& $\mathrm{G}$ & 6,62 & 5,61 & 1,66 & 43,43 & 5,72 & 0,65 & 0,25 & $-0,61$ \\
& $\mathrm{~T}$ mineral $\left(\mathrm{mg} \mathrm{kg}^{-1}\right.$ ) & 63,2 & 59,1 & 23,1 & 251,3 & 28,4 & 1,8 & 0,31 & 0,27 \\
& $\mathrm{G}$ & 59,9 & 56,3 & 25,9 & 136,5 & 25,17 & 2,85 & 0,04 & $-0,81$ \\
S disponible $\left(\mathrm{mg} \mathrm{kg}^{-1}\right)$ & $\mathrm{T}$ & 6,63 & 4,76 & 0,28 & 31,70 & 5,94 & 0,38 & 0,22 & $-0,24$ \\
& $\mathrm{G}$ & 6,58 & 4,57 & 0,52 & 31,70 & 5,87 & 0,66 & 0,60 & 0,78 \\
Suma bases $\left(\mathrm{cmol}_{+} \mathrm{kg}^{-1}\right)$ & $\mathrm{T}$ & 3,61 & 3,30 & 0,38 & 10,57 & 1,91 & 0,12 & $-0,26$ & $-0,11$ \\
& $\mathrm{G}$ & 3,54 & 3,21 & 0,43 & 10,57 & 2,09 & 0,24 & $-0,20$ & $-0,02$ \\
CICE $\left(\mathrm{cmol}_{+} \mathrm{kg}^{-1}\right.$ ) & $\mathrm{T}$ & 4,26 & 4,49 & 0,92 & 11,26 & 1,83 & 0,12 & $-0,22$ & 0,20 \\
& $\mathrm{G}$ & 4,20 & 3,71 & 0,92 & 11,26 & 2,14 & 0,24 & $-0,04$ & 0,19 \\
Saturación Al (\%) & $\mathrm{T}$ & 17,1 & 19,5 & 0,18 & 83,71 & 19,10 & 1,23 & 0,02 & $-1,09$ \\
& $\mathrm{G}$ & 17,7 & 8,00 & 0,25 & 78,08 & 19,96 & 2,26 & 0,00 & $-1,11$ \\
\hline
\end{tabular}

DE, desviación estándar de la media; EE, error estándar de la media; Sk, skewness; Kr, kurtosis. 
Cuadro 2. Resultados del análisis de variabilidad espacial para el transecto-T ( $n=242)$ y la grilla-G $(n=78)$.

Table 2. Results of spatial variability analysis for the transect-T $(n=242)$ and the grid-G $(n=78)$.

\begin{tabular}{|c|c|c|c|c|c|c|}
\hline Variable & Unidad & Modelo & $\mathbf{R}^{2}$ & $\mathrm{C} /(\mathrm{Co}+\mathrm{C})$ & $\begin{array}{c}\text { Rango } \\
\text { (m) }\end{array}$ & $\begin{array}{c}\text { Dependencia } \\
\text { espacial }\end{array}$ \\
\hline \multirow[t]{2}{*}{$\mathrm{pH} \mathrm{H} \mathrm{H}_{2} \mathrm{O}$} & $\mathrm{T}$ & ID & & & & \\
\hline & G & ID & & & & \\
\hline \multirow[t]{2}{*}{$\mathrm{pH} \mathrm{CaCl}_{2}$} & $\mathrm{~T}$ & ID & & & & \\
\hline & G & ID & & & & \\
\hline \multirow[t]{2}{*}{ P-Olsen } & $\mathrm{T}$ & Exponencial & 0,44 & 0,52 & 1146 & M \\
\hline & G & Esférico & 0,71 & 0,95 & 159 & $\mathrm{~F}$ \\
\hline \multirow[t]{2}{*}{$\mathrm{N}$ mineral } & $\mathrm{T}$ & Esférico & 0,35 & 0,50 & 605 & M \\
\hline & G & Gaussiano & 0,96 & 0,81 & 763 & $\mathrm{~F}$ \\
\hline \multirow[t]{2}{*}{ S disponible } & $\mathrm{T}$ & Exponencial & 0,50 & 0,65 & 2733 & M \\
\hline & G & Exponencial & 0,27 & 0,50 & 128 & M \\
\hline \multirow[t]{2}{*}{ Suma Bases } & $\mathrm{T}$ & Exponencial & 0,60 & 0,43 & 357 & $\mathrm{M}$ \\
\hline & & Esférico & 0,93 & 0,64 & 664 & M \\
\hline \multirow[t]{2}{*}{ CICE } & $\mathrm{T}$ & Lineal & 0,32 & 0,17 & 1900 & D \\
\hline & G & Gaussiano & 0,74 & 0,50 & 719 & M \\
\hline \multirow[t]{2}{*}{ Saturación de Al } & $\mathrm{T}$ & Exponencial & 0,90 & 0,50 & 1270 & M \\
\hline & G & ID & & & & \\
\hline
\end{tabular}

Nota: ID, variación es independiente de la distancia, interpolación por método inverso a la distancia; C/(C+Co), proporción de la varianza de la muestra $(\mathrm{C}+\mathrm{Co})$ que es explicada por la varianza espacialmente estructurada (C). Tipo de dependencia espacial según Cambardella y Karlen (1999): $\mathrm{F}$ es fuertemente dependiente, $\mathrm{M}$ es moderadamente dependiente y D, es débilmente dependiente.

Note: ID, variation is independent of the distance, inverse distance weighting interpolation; $\mathrm{C} /(\mathrm{C}+\mathrm{Co})$, ratio of the sample variance $(\mathrm{C}+\mathrm{Co})$ that is explained by the spatially structured variance (C). Type of spatial dependence according to Cambardella and Karlen (1994): F is strongly dependent and $\mathrm{M}$ is moderately dependent and $\mathrm{D}$ is weakly dependent.

la distancia, lo que es mostrado porque sus valores del semivarianza no fueron posibles de ser ajustados a ningún modelo, al menos en las escalas de observación estudiadas (Trangmar et al., 1985; Webster y Oliver, 2001), por lo cual, sería recomendable en futuros estudios con estas variables, considerar distancias menores de separación para la colecta de muestras, ya que a la escala evaluada no fue posible detectar un patrón de variación claro. Considerando lo anterior, para una misma propiedad, al variar la escala de muestreo, cambia el modelo y el tipo de dependencia espacial (Cambardella y Karlen, 1999). La Figura 3 presenta la distribución espacial de todas las propiedades químicas evaluadas. En ellas se muestra las variaciones espaciales, que se hacen evidentes en este análisis y que se pierden al considerar los valores promedios en las propiedades del suelo evaluadas.

Se evaluó la variación estacional del $\mathrm{pH}\left(\mathrm{H}_{2} \mathrm{O}\right.$ y CaCl 2$)$, $\mathrm{N}$ mineral y $\mathrm{S}$ disponible. Los valores de $\mathrm{N}$ mineral variaron entre 18,2-68,6 $\mathrm{mg} \mathrm{kg}^{-1}$, el S disponible entre 1,5-17,3 mg kg ${ }^{-1} \mathrm{y}$, los valores de $\mathrm{pH} \mathrm{H}_{2} \mathrm{O}$ y $\mathrm{CaCl}_{2}$, entre 4,51-6,03 y 3,86-5,29, respectivamente. Independiente del uso del suelo (pradera o bosque), se presentaron diferencias $(p<0,05)$ entre las fechas de muestreo; y para el S disponible y el $\mathrm{pH} \mathrm{CaCl}_{2}$ se detectaron diferencias entre los usos (Figura 4). Estas variaciones son relevantes y muestran diferencias en las estaciones del año. Las mayores variaciones estacionales fueron para el $\mathrm{N}$ mineral, entre invierno y primavera. Incluso, se determinó variación para la misma estación pero entre años, como primavera entre los años 2011 y 2012. Lo anterior, podría estar asociado a cambios en la mineralización de $\mathrm{N}$ del suelo, los cuales a su vez, se asocian con las variaciones climáticas, principalmente temperatura y precipitación (Ross, 1987; Corre et al., 2002). Las mayores diferencias entre los usos de pradera y bosque se dieron para la variable de $\mathrm{pH}$, no así para $\mathrm{N}$ y S, lo cual podría estar asociado a los ingresos de material orgánico al suelo. Buytaert et al. (2007), encontraron pocas diferencias de sus variables químicas dadas por los usos, lo cual atribuyen al poco tiempo de utilización de sus suelos volcánicos. $\mathrm{El} \mathrm{pH} \mathrm{CaCl}_{2}$, resultó ser una variable más estable entre las estaciones evaluadas (Figura 4), esta cualidad en la determinación del $\mathrm{pH} \mathrm{CaCl}_{2}$ es ventajo- 
sa desde el punto de vista de la fertilidad de los suelos y posibles recomendaciones a partir de ella, ya que podría independizar la determinación de la estación del año. Estas variaciones estacionales debieran ser consideradas cuando se diseñan las prácticas de manejo, para no sub o sobreestimar los aportes del sistema suelo (Haines y Cleveland, 1981). Considerando todos los datos (transecto, grilla y estaciones), se obtuvo una estrecha relación lineal

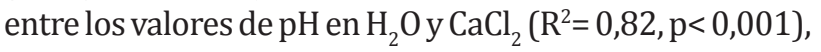
al igual que para la relación, de tipo exponencial negativa, entre $\mathrm{pH}$ y saturación de $\mathrm{Al}\left(\mathrm{R}_{\text {agua }}^{2}=0,63\right.$ y $\mathrm{R}_{\text {cloruro calcio }}=0,77$, $\mathrm{p}<0,001)$, similar a la relación mostrada por Rowell (1994) y Charman y Murphy (2000). La mejor estimación de la saturación de $\mathrm{Al}$ con el $\mathrm{pH} \mathrm{CaCl}{ }_{2}$ que en $\mathrm{H}_{2} \mathrm{O}$, reforzarían la idea de considerar en el futuro esta determinación de $\mathrm{pH}$ para planes de manejo.

a) $\mathrm{pH} \mathrm{H}_{2} \mathrm{O}(1: 2.5)$
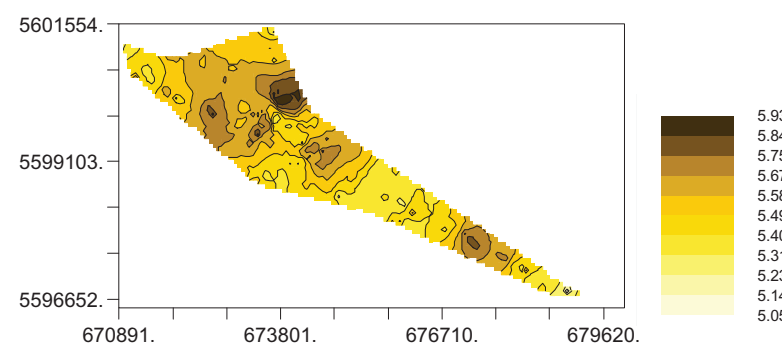

b) P-Olsen

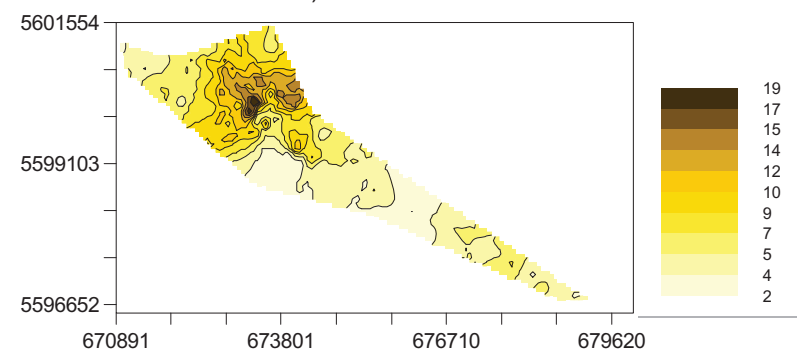

c) $\mathrm{N}$ mineral

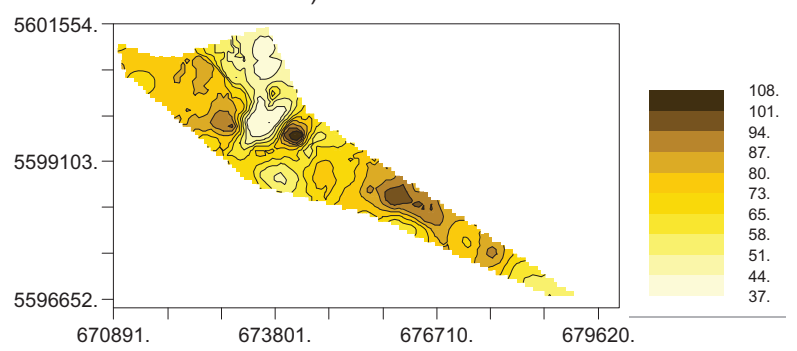

\section{CONCLUSIONES}

En este estudio se evaluó la variabilidad espacial y temporal de algunas propiedades químicas asociadas a la disponibilidad de nutrientes y de la condición de acidez en suelos chilenos de origen volcánico. De los resultados encontrados, se puede concluir, que cada una de las propiedades químicas presentó diferentes tipos de variabilidad, que es influida por la escala de observación. Ello muestra, que esta variabilidad se debe considerar al diseñar nuevas prácticas de manejo para cada uso de suelo. Es por ello, que se hace evidente no sólo considerar los promedios de los atributos químicos para un sector determinado, sino su distribución espacial y temporal, ya que pueden variar significativamente en función de la época del año.
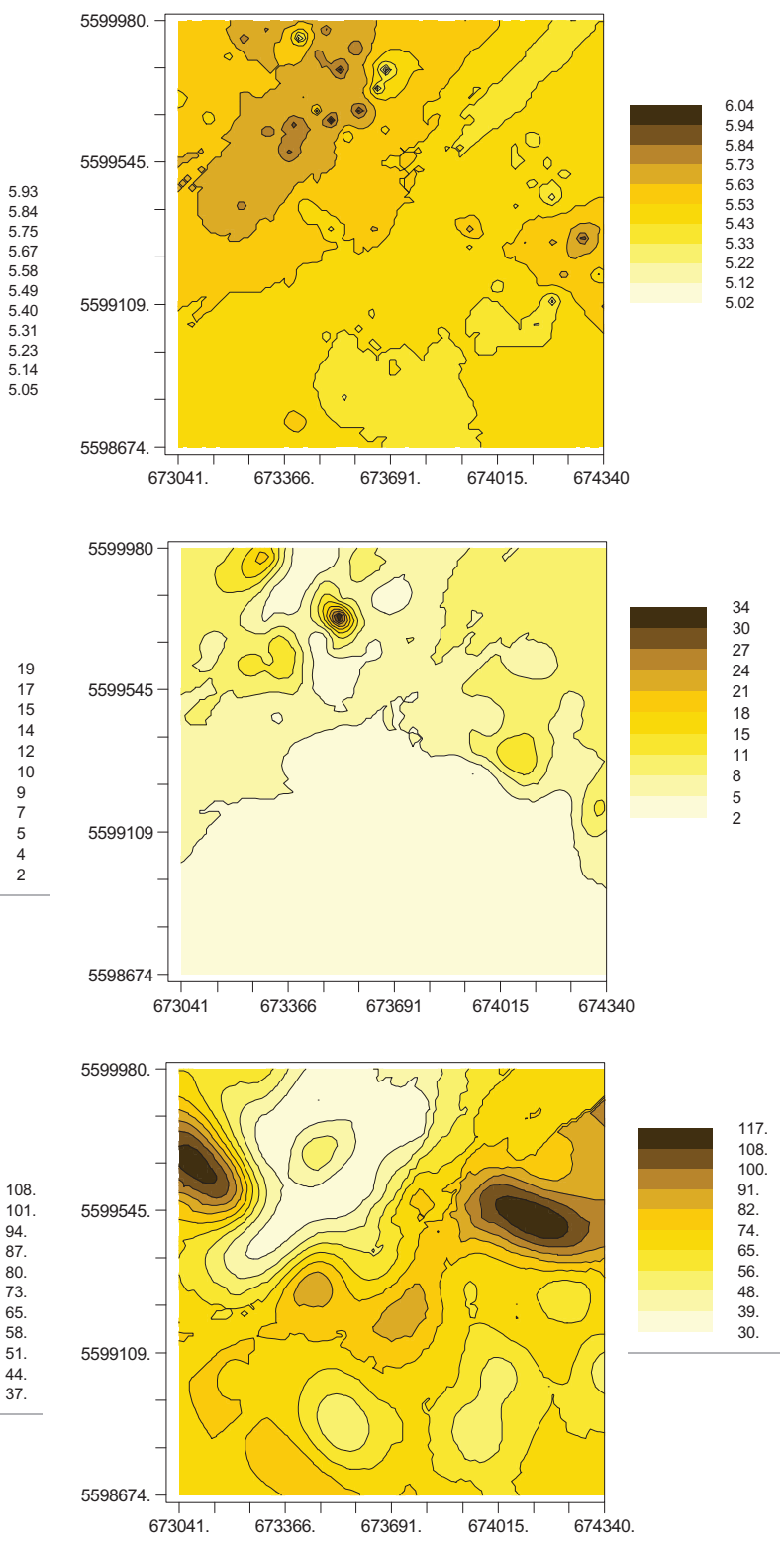

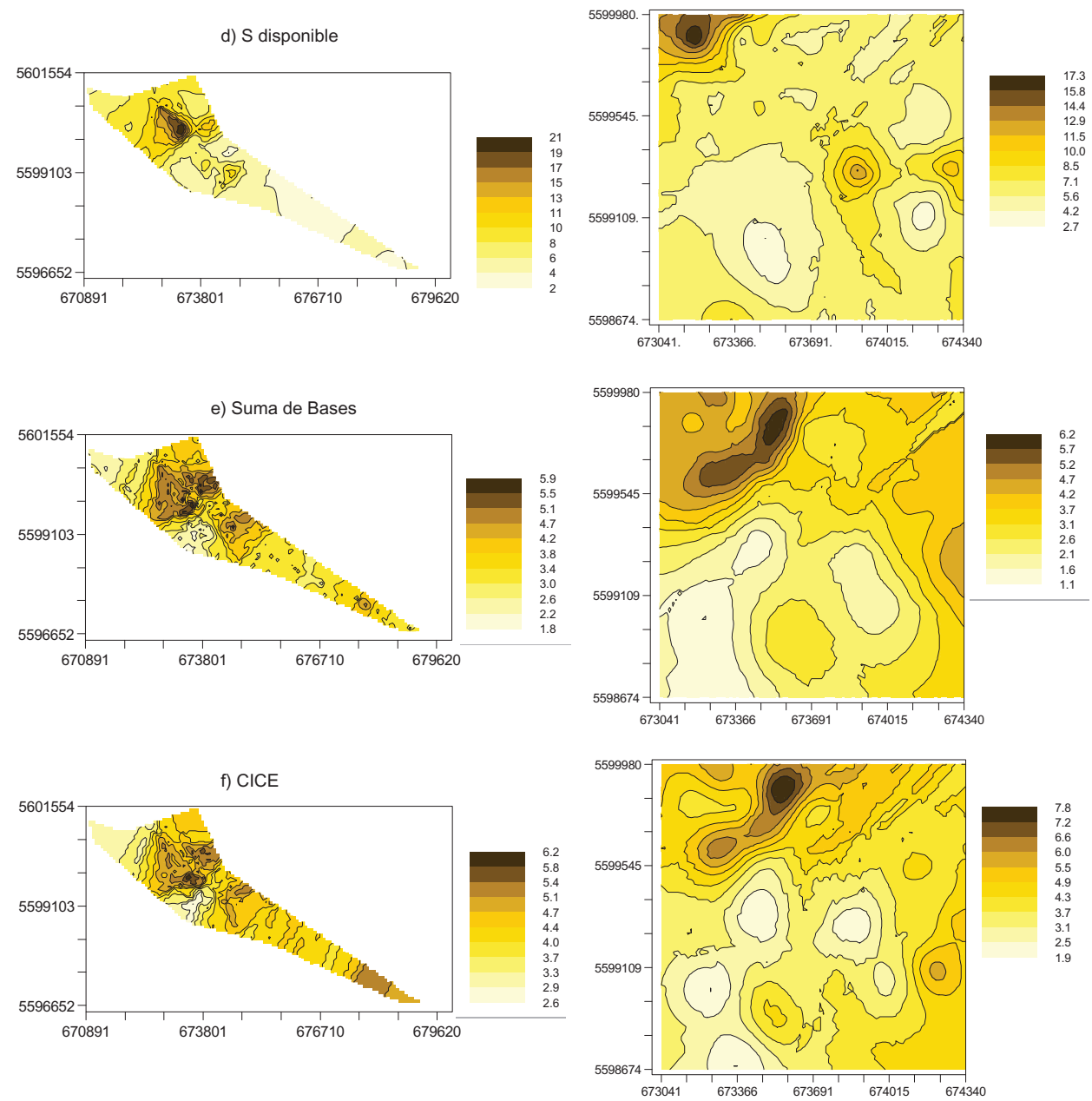

Figura 3. Variabilidad espacial del a) $\mathrm{pH}-\mathrm{H}_{2} \mathrm{O}$, b) P-Olsen, c) $\mathrm{N}$ mineral, d) S disponible, e) suma de bases y f) CICE. Figuras a la izquierda corresponden al muestreo de suelos en el transecto, y a la derecha a la grilla.

Figure 3. Spatial variability of a) $\mathrm{pH}-\mathrm{H}_{2} \mathrm{O}$, b) P-Olsen, c) mineral $\mathrm{N}$, d) available $\mathrm{S}$, e) base sum and f) ECEC. Figures to the left correspond to the soil sampling transect, and to the right the grid.
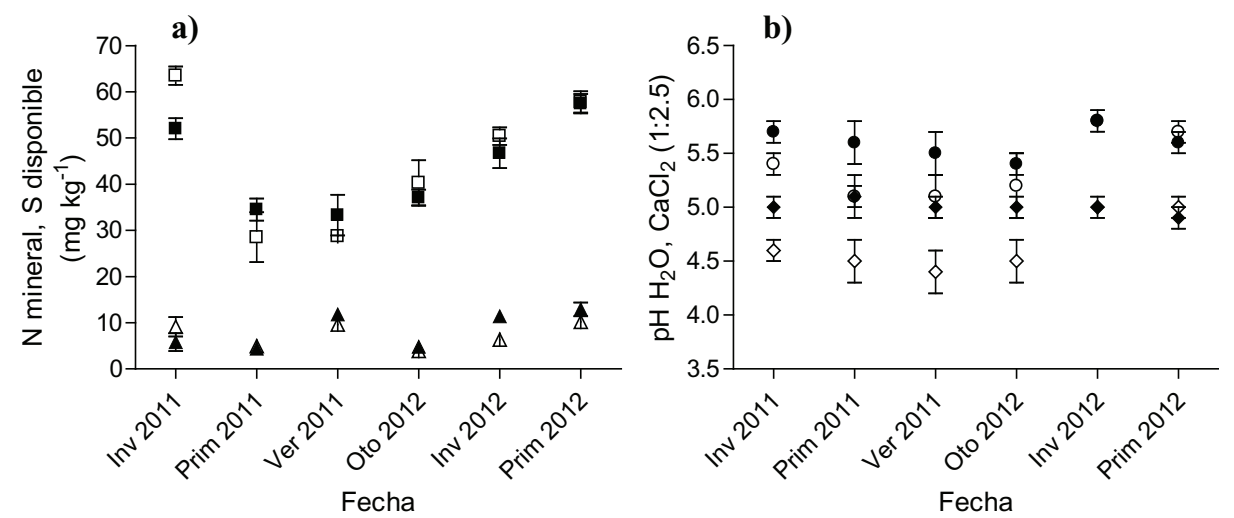

Figura 4. Variación estacional del suelo bajo pradera (símbolos llenos) y bosque (símbolos vacíos), para las propiedades de: a) $\mathrm{N}$ mineral (cuadrados) y $\mathrm{S}$ disponible (triángulos) y b) pH en $\mathrm{H}_{2} \mathrm{O}$ (círculos) y en $\mathrm{CaCl}_{2}$ (rombos). Barras verticales muestran el error estándar de la media.

Figure 4. Soil seasonal variation under grassland (filled symbols) and forest (open symbols) for the following properties: a) mineral $\mathrm{N}$ (squares) and available $\mathrm{S}$ (triangles) and, b) $\mathrm{pH}$ in $\mathrm{H}_{2} \mathrm{O}$ (circles) and $\mathrm{CaCl}_{2}$ (diamonds). Vertical bars show the standard error of the mean. 


\section{REFERENCIAS}

BERNIER, R. 2000. Análisis de Suelo. Metodología e Interpretación. Serie Actas №2. INIA-Remehue.

BERTRAND, S., FAGEL, N. 2008. Nature, origin, transport and deposition of andosol parent material in south-central Chile (36-42 $\left.{ }^{\circ}\right)$. Catena 73: 10-22.

BUYTAERT, W., DECKERS, J., WYSEURE, G. 2007. Regional variability of volcanic ash soils in south Ecuador: The relation with parent material climate and use. Catena 70: 143-154.

BESOAIN, E. 1985. Mineralogía de los suelos volcánicos del centro sur de Chile. In: Tosso, J. (Ed.). Suelos Volcánicos de Chile. Instituto de Investigaciones Agropecuarias, Chile (INIA). pp. 109-151.

BURROUGH, P.A. 1993. Soil variability: a late 20th century view. Soils and Fertilizers. 56: 529-562.

CAMBARDELLA, C.A., MOORMAN, T.B., NOVAK, J.M., PARKIN, T.B., KARLEN, D.L., TURCO, R.F., KONOPKA, A.E. 1994. Field scale variability of soil properties in central Iowa soils. Soil Science Society of American Journal 58: 1501-1511.

CAMBARDELLA, C.A., KARLEN, D.L. 1999. Spatial analysis of soil fertility parameters. Precision Agriculture 1: 5-14.

CHARMAN, P.E.V., MURPHY, B.W. 2000. Soils: Their properties and management. Oxford University Press, Oxford.

CORRE, M.D., SCHNABEL, R.R., STOUT, W.L. 2002. Spatial and seasonal variation of gross nitrogen transformations and microbial biomass in an Northeastern US grassland. Soil Biology and Biochemistry 34: 445-457.

DAHLGREN, R.A., SAIGUSA, M., UGOLINI, F.C. 2004. The nature, properties and management of volcanic soils. Advances in Agronomy 82: 113-182.

GOOVAERTS, P. 1998. Geostatistical tools for characterizing the spatial variability of microbiological and physic-chemical soil properties. Biology and Fertility of Soils 27: 315-334.

HAINES, S.G., CLEVELAND, G. 1981. Seasonal variation in properties of five forest soils in southwest Georgia. Soil Science Society of American Journal 45(1): 139-143.

JENNY, H. 1941. Factors of Soil Formation. A System of Quantitative Pedology. McGraw-Hill, New York.

LOGSDON, S.D. 2012. Temporal variability of bulk density and soil water at selected field sites. Soil Science 177: 327-331.

NIELSEN, D.R., WENDROTH, O. 2003. Spatial and Temporal Statistics. Sampling Field Soils and Their Vegetation.
Catena Verlag, Reiskirchen.

PARKIN, T.B. 1993. Spatial variability of microbial process in a soil - a review. Journal of Environmental Quality 22: 409-417.

ROSS, D.J. 1987. Soil microbial biomass estimated by the fumigation-incubation procedure: Seasonal fluctuations and influence of soil moisture content. Soil Biology and Biochemistry 19: 397-404.

ROWELL, D.L. 1994. Soil Science: Methods and Applications. Taylor \& Francis, London, New York.

SADZAWKA, A., CARRASCO, M.A., GREZ, R., MORA, M.L. 2004. Métodos de análisis recomendados para suelos de chilenos. Comisión de Normalización y Acreditación (CNA). Sociedad Chilena de la Ciencia del Suelo. Santiago.

SADZAWKA, A., CARRASCO, M.A., GREZ, R., MORA, M.L. 2006. Acidificación de los suelos volcánicos de Chile. In: http://www2.inia.cl/medios/Descargas/CRI/Platina/ ResumenesCongresos/2006/2006-001-sadzawka.pdf

SIMONSON, R.W. 1959. Outline of a generalized theory of soil genesis. Soil Science Society of American Procedures 23: 152-156.

SHOJI, S., NANZYO, M., DALHLGREN, R. 1993. Volcanic ash soils, properties and utilization. Developments in soil science. Elsevier Science. Amsterdam, New Zealand.

SPOSITO, G. 2008. The Chemistry of Soils. Oxford University Press, Oxford, New York.

TIMM, L. C., PIRES, L. F., ROVERATTI, R., ARTHUR, R.C.J., REICHARDT, K., MARTINS DE OLIVEIRA, J.C., BACCHI, O.O.S. 2006. Field spatial and temporal patterns of soil water content and bulk density changes. Scientia Agricola 63: 55-64.

TRANGMAR, B.B., YOST, R.S., UEHARA, G. 1985. Application of geostatistics to spatial studies of soil properties. Advances in Agronomy 38: 45-94.

WEBSTER, S., OLIVER, M. 1990. Statistical Methods in soil and land resource survey. Spatial information system. Oxford University, New York

WEBSTER, R., OLIVER, M. 2001. Geostatistics for Environmental Scientists. John Wiley \& Sons, Ltd. Chichester.

YEMEFACK, M., ROSSITER D.G., NJOMGANG, R. 2005. Multiscale characterization of soil variability within an agricultural landscape mosaic system in southern Cameroon. Geoderma 125: 117-143.

ZHANG, X.Y., SUI, Y.Y., ZHANG, X.D., MENG, K., HERBERT, S.J. 2007. Spatial variability of nutrient properties in black soil of Northeast China. Pedosphere 17: 19-29. 